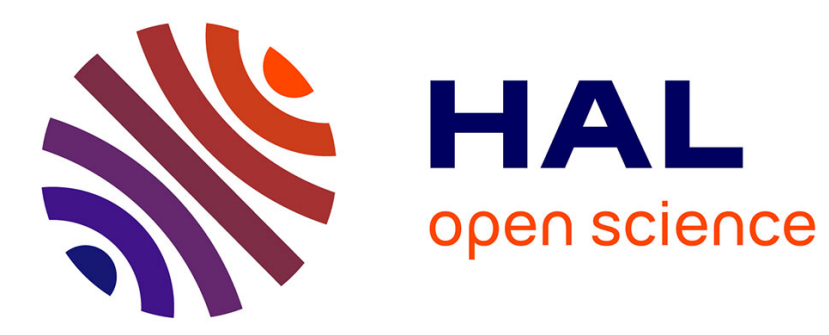

\title{
A Note on the Chas-Sullivan product
}

Francois Laudenbach

\section{- To cite this version:}

Francois Laudenbach. A Note on the Chas-Sullivan product. L'Enseignement Mathématique, 2011, 2 (57), pp.1-19. hal-00368536v2

\section{HAL Id: hal-00368536 https://hal.science/hal-00368536v2}

Submitted on 11 Feb 2010

HAL is a multi-disciplinary open access archive for the deposit and dissemination of scientific research documents, whether they are published or not. The documents may come from teaching and research institutions in France or abroad, or from public or private research centers.
L'archive ouverte pluridisciplinaire HAL, est destinée au dépôt et à la diffusion de documents scientifiques de niveau recherche, publiés ou non, émanant des établissements d'enseignement et de recherche français ou étrangers, des laboratoires publics ou privés. 


\title{
A note on the Chas-Sullivan product
}

\author{
FRANÇOIS LAUDENBACH
}

\begin{abstract}
We give a finite dimensional approach to the Chas-Sullivan product on the free loop space of a manifold, orientable or not, compact or not.
\end{abstract}

\section{INTRODUCTION}

Let $M$ be an $n$-dimensional manifold with empty boundary; it is not required to be either compact or orientable. Denote $L M=C^{\infty}\left(S^{1}, M\right)$ be its free loop space. In a famous paper [⿴囗十), in the case when $M$ is an orientable and closed manifold, M. Chas and D. Sullivan constructed a natural graded algebra structure on the homology $H_{*}(L M ; \mathbb{Z})$, more precisely a product

$$
H_{i}(L M ; \mathbb{Z}) \otimes H_{j}(L M ; \mathbb{Z}) \rightarrow H_{i+j-n}(L M ; \mathbb{Z}),
$$

in the same intersection spirit as the usual intersection product on $H_{*}(M ; \mathbb{Z})$. But their ideas remained not completely accomplished. A different approach has been considered by $\mathrm{R}$. Cohen and J. Jones in [6]. According to their abstract, they describe "a realization of the ChasSullivan product in terms of a ring spectrum structure on the Thom spectrum of a certain virtual bundle over the loop space", a difficult technique indeed. Recently in [8], Y. Félix and J.-C. Thomas have put String Topology into a broad homotopy theoretical setting; they prove that the operations in string topology are preserved by homotopy equivalence, at least in the 1connected case. On the contrary, in the present note we propose a finite dimensional approach, very close to the spirit of [1], based on transversality arguments. We also treat the case of a non-orientable manifold using local coefficients instead of $\mathbb{Z}$.

We do not think of $L M$ as a topological space but as a simplicial set (except in section 4 ). A $k$-simplex in $L M$ is a smooth map $\Sigma: \Delta^{k} \times S^{1} \rightarrow M$ where $\Delta^{k}$ is the standard $k$-simplex. Let 0 be the base point of $S^{1}=\mathbb{R} / \mathbb{Z}$. The evaluation map $e v_{0}: L M \rightarrow M$ is simplicial: $e v_{0}(\Sigma)=\sigma$ where $\sigma: \Delta^{k} \rightarrow M$ is the $k$-simplex of $M$ defined by

$$
\sigma(t)=\Sigma(t, 0) \text {. }
$$

It is easy to form a chain complex based on the simplices of $L M$ and a bi-complex based on bi-simplices (pairs of simplices). In order to take into account the non-orientabiliy, we limit ourselves to small simplices and bi-simplices (see 2.1). To define an intersection product we consider transverse bi-simplexes. By Thom's transversality theorem, they generate a sub-bicomplex which has the same homology as $L M \times L M$. As we shall see, smallness (resp. transversality property) will only refer to the image of simplices (resp. bi-simplices) through the evaluation

2000 Mathematics Subject Classification. 55N10, 57R19, 57R45.

Key words and phrases. Free loop space, intersection product, Chas-Sullivan product. 
map. Let us introduce the sub-bicomplex $L M \underset{M}{\times} L M$ of $L M \times L M$ made of composable loops, that is, pairs of loops having a common origin. Performing the composition yields a well defined map in homology $L M \underset{M}{\times} M \rightarrow L M$ The intersection product will be not far from being defined on the chain level: the intersection of two transverse cycles in $L M$ will produce a "singular" smooth manifold $W$ in $L M \underset{M}{\times} L M$, hence in $L M$ after composing, which becomes a simplicial cycle once $W$ is triangulated. According to Whitehead [13], such a triangulation is unique up to subdivision and isotopy. Therefore the product is well defined at the homology level.

In last section it is shown that this definition of the free loop product is not less efficient than the "infinite dimensional" one when calculations are performed in concrete geometric situations. Results due to different authors (M. Goresky, N. Hingston in [9], D. Chataur, J.-F. Le Borgne in [5]) are rewritten in this setting.

ACKnowledgments. I had the chance to hear Dennis Sullivan lecturing on this subject on the occasion of his Doctorat Honoris Causa at École normale supérieure (Lyon) in Dec. 2001. Later on, I had fruitful conversations with David Chataur and Hossein Abbaspour who gave me more details. I feel indebted to all of them. I am also grateful to Jean-Claude Thomas for comments on a preliminary version of this note and to Antoine Touzé who prevented me from making a mistake in spectral sequences.

\section{Simplices AND BI-Simplices AT THE MANifold LEVEL}

In this section we give a geometrical approach to the intersection product in the homology of $M$. The important point is that it is done in a way so that the intersection product lifts immediatly to $L M$. This requires an appeal to Thom's transversality with constraints which is more powerful than the usual transversality, as it is usually done (see J.E. McClure [11] in the $P L$ case or E. Castillo, R. Diaz [3] in the smooth case).

2.1. The manifold $M$ under consideration is equipped with an atlas $\mathcal{A}$ of charts. A small $k$-simplex is a smooth map

$$
\sigma: \Delta^{k} \rightarrow M
$$

whose image is contained in a chart from $\mathcal{A}$. For each simplex $\sigma$ some particular chart $U(\sigma) \in \mathcal{A}$ is chosen once for all containing its image. A small $k$-chain is a linear combination $\xi=\sum n_{i} \sigma_{i}$ with coefficient $n_{i} \in \mathbb{Z}$ of finitely many small simplices $\sigma_{i}$. The oriention twisted boundary is defined by the following formula:

$$
\partial \sigma=\sum_{i=0}^{k} \varepsilon(-1)^{i} F_{i} \sigma,
$$

where $F_{i} \sigma$ is the $i^{\text {th }}$ face of $\sigma$ and $\varepsilon$ is the sign of the Jacobian of change of coordinates from $U\left(F_{i} \sigma\right)$ to $U(\sigma)$ calculated at any point of the image of $F_{i} \sigma$. The small chains with this boundary form a chain complex whose homology is $H_{*}\left(M ; \mathbb{Z}_{o r}\right)$, the homology with integral coefficients twisted by the orientation. 
In the sequel, homotopy means smooth homotopy. Given a $k$-simplex $\sigma$, a homotopy $\sigma^{t}$ : $\Delta^{k} \rightarrow M, t \in[0,1]$, with $\sigma^{0}=\sigma$ induces a homotopy $(F \sigma)^{t}$ of each face $F \sigma$. So the following definition makes sense.

Definition 2.2. Given a chain $\xi=\sum n_{i} \sigma_{i}$, a boundary preserving homotopy of $\xi$ is a one parameter family $\xi^{t}, t \in[0,1], \xi^{t}=\sum n_{i} \sigma_{i}^{t}$, where $t \mapsto \sigma_{i}^{t}$ is a homotopy of $\sigma_{i}$ into $M\left(\sigma_{i}^{0}=\sigma_{i}\right)$, and such that, if $\sigma_{i}$ and $\sigma_{j}$ have a common face at time $t=0$ the corresponding simplices $\sigma_{i}^{t}$ and $\sigma_{j}^{t}$ still have a common face at any time.

For instance, if $\xi$ is a cycle then $\xi^{t}$ is a cycle for every $t \in[0,1]$. When we consider such a homotopy of small chain, we shall limit ourselves to the case when the homotopy is small, that is: each summand $\sigma_{i}^{t}$ takes place in $U\left(\sigma_{i}\right)$. The following homotopy extension lemma is easy to prove.

Lemma 2.3. Let $\tau$ be any simplex which is a face of one of the summands of $\xi$. Any (small) homotopy of $\tau$ extends as a (small) boundary preserving homotopy of $\xi$. The same statement holds for a family of homotopies with an extension depending continuously on the parameter.

Definition 2.4. $A(p, q)$-bi-simplex of $M \times M$ is a pair $(u, v)$ where $u$ is a p-simplex and $v$ is a q-simplex of $M$. It is said to be small when both factors are small simplices of $M$.

It is convenient to denote it $u \times v$ like the map $u \times v: \Delta^{p} \times \Delta^{q} \rightarrow M \times M,(x, y) \mapsto(u(x), v(y))$. The (small) bi-simplices generate a bi-complex $C_{* *}(M \times M)$ whose elements are bi-chains, with two boundary operators twisted by the orientation,

$$
\partial_{1}(u \times v)=(\partial u) \times v, \partial_{2}(u \times v)=u \times \partial v,
$$

and a total boundary operator

$$
D(u \times v)=\partial u \times v+(-1)^{p} u \times \partial v .
$$

Definition 2.5. A (small) bi-simplex $u \times v$ is said to be transverse when the map $u \times v$ is transverse to the diagonal $\Delta_{M}$ and when all its faces (they are bi-simplices) are also transverse to the diagonal.

In the sequel, all transverse bi-simplices will be small, without saying it. The advantage is that $W=(u \times v)^{-1}\left(\Delta_{M}\right)$ is a proper orientable submanifold of codimension $n$ (with corners) in $\Delta^{p} \times \Delta^{q}$. Moreover, if we use the charts $U(u)$ and $U(v)$ whose product contains the image of $u \times v, W$ receives a canonical orientation.

Notice that, when $u \times v$ is a transverse bi-simplex, a small homotopy of one of the factors keeps them transverse. The transverse bi-simplices generate a sub-bicomplex $C_{* *}^{\text {tr }}(M \times M)$ of $C_{* *}(M \times M)$. A bi-chain is said to be transverse when each of its bi-simplices is transverse. It is said to be a product bi-chain if it has the form $\xi \times \eta$ where both factors are chains in $M$.

Lemma 2.6. Let $\xi \times \eta$ be a product bi-chain. There exists an arbitrarily $C^{\infty}$-small boundary preserving homotopy $\xi^{t}$ of $\xi^{0}=\xi$ such that $\xi^{1} \times \eta$ is transverse. Moreover, when $\partial \xi \times \eta$ is transverse, the homotopy can be chosen stationary on $\partial \xi \times \eta$. The same statement holds for the second factor. 
Considering the composition of loops we have in mind, it is very important to make this approximation among the bi-chains.

Proof. We first consider the case when $\xi \times \eta=u \times v$ is a bi-simplex. The conclusion follows from Thom's transversality theorem, not in its elementary form but in the form known as the transversality with constraint $([12])$. Indeed, only the first factor is moved for guaranteeing that bi-simplices remain bi-simplices during the deformation. We argue as follows. Let $S$ be a small $n$-ball in the vector space containing the chart $U(u)$, so small that, for any $s \in S, u^{s}:=u+s$ is still contained in $U(u)$. We introduce the family $\left(u^{s} \times v\right)$ parametrized by $S$. It is transverse to $\Delta_{M}$ as well as its restriction to any face of $\Delta^{p} \times \Delta^{q}$. Therefore, according to Sard's lemma (used à la Thom), for almost every $s \in S, u^{s} \times v$ is a transverse bi-simplex.

For the general case, it is useful to observe that, in the above argument, the space $S$ of parameters can be chosen arbitrarily small. When considering a product bi-chain $\xi \times \eta$, its $k$-skeleton is the collection of all the $k$-faces of the bi-simplices appearing in $\xi \times \eta$. Arguing recursively, we may assume that all bi-simplices in the $k$-skeleton are transverse. Let $(u \times v)$ be a $(k+1)$-bi-simplex, which we endow with a family $S$ of translations in $U(u)$. According to lemma 2.3, the translation by any $s \in S$ extends as a boundary preserving homotopy of $\xi$ (ending at $\xi_{s}$ ) and it can be chosen small enough so that each bi-simplex of the $k$-skeleton remains transverse. Therefore, for almost every $s \in S, \xi_{s}$ is transverse along its $k$-skeleton and $u \times v$. Repeating this process, we successively make all $(k+1)$-bi-simplices transverse.

For the relative version, we notice that, if $F u$ is a face of $u$ and $F u \times v$ is transverse, then $u \times v$ is automatically transverse to $\Delta_{M}$ on a neighborhood of the domain of $F u \times v$. In that case we can moderate the translation by $s$ so that it becomes stationary along $F u \times v$. The general relative version follows easily.

Let $[\xi]$ and $[\eta]$ be two classes of respective degrees $p$ and $q$ in $H_{*}\left(M ; \mathbb{Z}_{\text {or }}\right)$. According to lemma 2.6, they can be represented by small cycles so that the bi-cycle $\xi \times \eta$ is transverse. This bi-cycle is unique up to transverse homology; more precisely we claim the following uniqueness lemma:

Lemma 2.7. Let $\xi \times \eta$ and $\xi^{\prime} \times \eta^{\prime}$ be two transverse bi-cycles in the same bi-homology class $\left([\xi]=\left[\xi^{\prime}\right]\right.$ and $\left.[\eta]=\left[\eta^{\prime}\right]\right)$. There exists a transverse bi-chain $\Omega$ whose total boundary is $D \Omega=$ $\xi^{\prime} \times \eta^{\prime}-\xi \times \eta$.

Proof. There exists a cycle $\xi^{\prime \prime}$, homotopic to $\xi$, such that $\xi^{\prime \prime} \times \eta$ and $\xi^{\prime \prime} \times \eta^{\prime}$ are both transverse $(\xi \times \eta$ is already transverse and this property is preserved by approximation of $\xi$; according to lemma 2.6, such an approximation exists making $\xi^{\prime \prime} \times \eta^{\prime}$ transverse). If $\omega$ is a $(p+1)$-chain such that $\partial \omega=\xi-\xi^{\prime \prime}$, there is a boundary preserving homotopy $\omega^{t}, t \in[0,1]$, of $\omega$ relative to its boundary so that $\omega^{1} \times \eta$ is transverse. By the same argument there is a $(p+1)$-chain $\omega^{\prime}$ with $\partial \omega^{\prime}=\eta-\eta^{\prime}$, such that $\xi^{\prime \prime} \times \omega^{\prime}$ is transverse. Eventually, there exists $\omega^{\prime \prime}$ with $\partial \omega^{\prime \prime}=\xi^{\prime}-\xi^{\prime \prime}$ such that $\omega^{\prime \prime} \times \eta^{\prime}$ is transverse. By concatening the three transverse homologies $\omega^{1} \times \eta, \xi^{\prime \prime} \times \omega^{\prime}$ and $\omega^{\prime \prime} \times \eta^{\prime}$ we get a transverse homology joining both given bi-cycles.

2.8. Intersection of cycles. 
We are now ready for defining the intersection of cycles. To begin with, we consider a transverse product bi-chain $\xi \times \eta$ of degree $(p, q)$, which reads as a sum of transverse bi-simplices

$$
\xi \times \eta=\sum n_{i j} u_{i} \times v_{j} .
$$

Let $W_{i j}$ be the preimage of $\Delta_{M}$ by $u_{i} \times v_{j}$. As already noted, $W_{i j}$ is an oriented manifold with corners of codimension $n$. If $F\left(u_{i} \times v_{j}\right)$ is a face of the bi-simplex, $F W_{i j}$ denotes the corresponding face of $W_{i j}$.

According to Whitehead [13], $W_{i j}$ can be smoothly triangulated by a $P L$-triangulation $T_{i j}$. Moreover if some faces have been already triangulated, by using the relative version of Whitehead's theorem, $T_{i j}$ can be chosen so that the triangulated faces are subcomplexes. If two bi-simplices have common faces $F\left(u_{i} \times v_{j}\right)=F\left(u_{k} \times v_{\ell}\right)$, then we have a canonical diffeomorphism $F W_{i j} \rightarrow F W_{k \ell}$ which we think of as an identification. The triangulations of these faces are chosen accordingly. We consider the chain of $\Delta_{M} \cong M$

$$
\xi \cdot \eta:=(-1)^{n(n-q)} \sum n_{i j}\left(u_{i} \times v_{j}\right) \mid\left(W_{i j}, T_{i j}\right)
$$

which is called the intersection product. Of course, as a chain it depends on the chosen triangulations. But, since the latter are unique up to subdividing and boundary preserving isotopy (that is, smooth isotopy of each simplex in $W_{i j}$ keeping the triangulation property), the ambiguity is not severe. The sign, which we call the Dold sign, will be commented upon later.

Lemma 2.9. When $\xi \times \eta$ is a transverse bi-cycle, the intersection product $\xi \cdot \eta$ is a cycle of degree $p+q-n$ (with orientation twisted coefficients). If $\xi \times \eta$ is changed by a transverse homology (in the sense of lemma [2.7), $\xi \cdot \eta$ is changed by a homology. Finally, $[\xi] \cdot[\eta]$ is well-defined in $H_{p+q-n}\left(M ; \mathbb{Z}_{o r}\right)$.

Note that a change of triangulation of the $W_{i j}$ 's may be thought of as a special case of a change by a transverse homology.

Proof. As $W_{i j}$ is an oriented proper submanifold, $\left(W_{i j}, T_{i j}\right)$ is a relative cycle in $\Delta^{p} \times \Delta^{q}$. Thus, the total boundary of $\left(u_{i} \times v_{j}\right) \mid\left(W_{i j}, T_{i j}\right)$ is

$$
\left(\left(\partial u_{i}\right) \times v_{j}\right)\left|\left(W_{i j}, T_{i j}\right)+(-1)^{p}\left(u_{i} \times\left(\partial v_{j}\right)\right)\right|\left(W_{i j}, T_{i j}\right) .
$$

By summing on $i j$ we get the boundary of $\xi \cdot \eta$. As $\xi$ and $\eta$ are cycles, each hyperface in the latter sum appears twice with opposite sign. The rest of the statement is easy to prove.

Remark 2.10. In his book [7] (Chap. VIII, §13.3), A. Dold explains that the chosen sign makes the intersection product on homology and the (unsigned) cup-product on cohomology fit together via the Poincaré duality. Another advantage of this sign is the following. Set $\mathbb{H}_{*}\left(M ; \mathbb{Z}_{\text {or }}\right)=H_{*+n}\left(M ; \mathbb{Z}_{\text {or }}\right)$. This regraded homology endowed with the above intersection product becomes a commutative ring in the graded sense. 


\section{Simplices AND BI-Simplices AT THE FREE LOOP SPACE LEVEL}

3.1. We recall the evaluation map ev $: L M \rightarrow M$. A simplex $\Sigma: \Delta^{k} \times S^{1} \rightarrow M$ is said to be small when $\sigma=e v_{0}(\Sigma)$ is so. The $i^{t h}$-face of $\Sigma$ is is obtained by restricting $\Sigma$ to $F_{i} \Delta^{k} \times S^{1}$. We have $F_{i}\left(e v_{0}(\Sigma)\right)=e v_{0}\left(F_{i} \Sigma\right)$. The oriention twisted boundary of $\Sigma$ is

$$
\partial \Sigma=\sum_{i=0}^{k} \varepsilon(-1)^{i} F_{i} \Sigma,
$$

where $\varepsilon$ is the sign of the Jacobian of change of coordinates from $U\left(e v_{0}\left(F_{i} \Sigma\right)\right)$ to $U\left(e v_{0}(\Sigma)\right)$. The small chains with this boundary form a sub-complex of $C_{*}(L M)$, the singular chain complex of $L M$, whose homology is $H_{*}\left(L M ; \mathbb{Z}_{\text {or }}\right)$. Indeed, this sub-complex is obtained from $C_{*}(L M)$ by two operations which induce homotopy equivalences: subdivision and smoothing. The notion of boundary preserving homotopy is similar to the one given in 2.1 .

Lemma 3.2. The evaluation map has the lifting property for boundary preserving homotopy of chains with any initial chain. Moreover, if the lifting of the homotopy is given along some faces this partial lifting can be extended to a global lifting.

Proof. It is clear for $\Delta^{k} \times S^{1} \times[0,1]$ retracts onto $\Delta^{k} \times S^{1} \times\{0\} \cup \Delta^{k} \times\{0\} \times[0,1] \cup F \times S^{1} \times[0,1]$, where $F$ is any union of faces in $\Delta^{k}$.

A $(p, q)$-bi-simplex $u \times v$ in $L M \times L M$ is a map

$$
\begin{gathered}
\Delta^{p} \times \Delta^{q} \times S^{1} \rightarrow M \times M, \\
(x, y, \theta) \mapsto(u(x, \theta), v(y, \theta)) .
\end{gathered}
$$

It is said to be transverse when $\left(e v_{0}(u), e v_{0}(v)\right)$ is a transverse bi-simplex of $M \times M$. In that case, the preimage of the diagonal $\Delta_{M}$ yields a submanifold with corners $W \subset \Delta^{p} \times \Delta^{q}$. For each $(x, y) \in W$, the loops $u(x,-)$ and $v(y,-)$ are composable, as they have common base points $u(x, 0)=v(y, 0)$. Therefore, taking a triangulation of $W$ and the Dold sign as in 2.8, we get a $(p+q-n)$-chain of composable loops, which we call the loop intersection product:

$$
u * v:=(-1)^{n(n-q)}(u \times v) \mid W \times S^{1} .
$$

Performing the composition (in some order, $u$ before $v$ ) yields a $(p+q-n)$-chain in $L M$, called the Chas-Sullivan product or loop product $u_{C S} v$. Of course, it depends on the choice of the triangulation of $W$. Here, we see that the product structure of $u \times v$ is very important; without it we lose the entries of the composition. Thus, when making a bi-simplex transverse, it is crucial to do it by homotopy through bi-simplices. That is the reason why we used transversality with constraints.

This loop product extends linearly to the transverse bi-chains. When performing it on a product bi-cycle, the result is a cycle in $L M$ whose homology class in $H_{p+q-n}\left(L M ; \mathbb{Z}_{\text {or }}\right)$ is welldefined. Strictly speaking the system of coefficients is $e v_{0}^{*}\left(\mathbb{Z}_{\text {or }}\right)$, which we write $\mathbb{Z}_{\text {or }}$ for short and we shall do the same each time a loop space is in question. More precisely, we have the following proposition. 
Proposition 3.3. Let $[\xi] \in H_{p}\left(L M ; \mathbb{Z}_{o r}\right)$ and $[\eta] \in H_{q}\left(L M ; \mathbb{Z}_{\text {or }}\right)$. These classes can be represented by cycles in $L M$ such that $\xi \times \eta$ is a transverse bi-cycle. The class of $\xi{ }_{C S} \eta$ is uniquely defined in $H_{p+q-n}\left(L M ; \mathbb{Z}_{\text {or }}\right)$.

Proof. Starting with arbitrary representatives of the given homology classes in $L M$, lemma 2.6 is applied: it produces an homotopy of their images by the evaluation map making them a transverse bi-cycle in $M \times M$. The lifting homotopy property (lemma 3.2) allows one to make $\xi \times \eta$ a transverse bi-cycle in $L M \times L M$. For this representative, the loop product $\xi_{C S} \eta$ is well defined. If another representative $\xi^{\prime} \times \eta^{\prime}$ is used, one can prove that $\xi^{\prime} \times \eta^{\prime}$ and $\xi \times \eta$ are joined by a transverse homology, that is a transverse bi-chain in $L M \times L M$. It is nothing but a loop version of the uniqueness lemma 2.7 and it can be deduced from the latter by applying the lifting homotopy property. As a consequence the homology class of $\xi_{C S} \eta$ is well defined. $\diamond$

Remark 3.4. Of course the composition of smooth loops produces a piecewise smooth loop only. There are two ways for correcting this default. One consists of doing a smoothing (boundary preserving) homotopy. The other consists of using $L M=C^{0}\left(S^{1}, M\right)$ equipped with a mixed simplicial structure: a $k$-simplex will be a continuous map $u: \Delta^{k} \times S^{1} \rightarrow M$ whose restriction to $\Delta^{k} \times 0$ is smooth.

In the sequel we use the following simplified notation: $\xi \cdot \eta:=\xi_{C S} \eta$, which is defined when the bi-cycle $\xi \times \eta$ is transverse, and $[\xi] \cdot[\eta]:=\left[\xi_{C S} \eta\right]$ which is well defined. Actually, there is a 1-parameter family of compositions $-\cdot_{s}-, s \in[0,1]$, defined as follows. Two loops $u$ and $v$ are said to be $s$-composable when $u(s)=v(0)$; in that case the composed loop $u \cdot{ }_{s} v$ is made of $u|[0,1-s] * v * u|[1-s, 1]$. When $s=0$, it is the usual composition and when $s=1$ we have $u \cdot{ }_{1} v=v \cdot u$ (notice that if two loops are 0-composable, they are also 1-composable).

Proposition 3.5. At the homology level, the loop product is commutative up to sign. Precisely, if $\xi$ and $\eta$ are respectively a $p$-cycle and a q-cycle of $L M$, then $[\xi] \cdot[\eta]=(-1)^{(p-n)(q-n)}[\eta] \cdot[\xi]$. The loop product is also associative.

If the regrading $\mathbb{H}_{*}=H_{*+n}$ is applied, then $\mathbb{H}_{*}\left(L M, \mathbb{Z}_{o r}\right)$ when endowed with the loop product becomes a graded commutative and associative ring.

Proof. We assume that the bi-cycle $\xi \times \eta$ in $L M \times L M$ is transverse. We first prove:

$$
[\xi] \cdot[\eta]=[\xi \cdot 1 \eta] .
$$

Writing $\xi \times \eta=\sum n_{i j} u_{i} \times v_{j}$, one can make a boundary preserving homotopy of $\xi$ so that, for every $i j$, the following map

$$
(x, y, s) \in \Delta^{p} \times \Delta^{q} \times[0,1] \mapsto\left(u_{i}(x, s), v_{j}(y, 0), s\right) \in M \times M \times[0,1]
$$

is transverse to $\Delta_{M} \times[0,1]$. This transversality yields us a $(p+q-n+1)$-chain $\omega$ in $L M$ whose boundary is $\partial \omega=\xi \cdot 1 \eta-\xi \cdot \eta$. Note that the loops in $\omega$ have $u(x, 0)$ as base points; therefore, even if the loops in $u$ are orientation reversing no sign appears in the formula of $\partial \omega$.

Now, we are reduced to proving:

$$
[\xi \cdot 1 \eta]=(-1)^{(p-n)(q-n)}[\eta] \cdot[\xi] .
$$


On both sides of this equality the composition is the same. The only difference comes from the orientation of the manifold $W_{i j}$ associated to each transverse bi-simplex $u_{i} \times v_{j}$ appearing in $\xi \times \eta$. The permutation of both factors in this bi-simplex induces a change $(-1)^{n}$ in the co-orientation of the diagonal $\Delta_{M}$ and a change $(-1)^{p q}$ due to the order of the factors $\Delta^{p}$ and $\Delta^{q}$ in the source. Moreover, the Dold sign $(-1)^{n(n-q)}$ is changed to $(-1)^{n(n-p)}$. Altogether, the sign rule follows.

For associativity, if three chains $\xi, \eta, \zeta$ (of respective degrees $p, q, r$ ) are given in $L M, \xi \times \eta \times \zeta$ is easily defined to be transverse. If they are cycles and if the triple is transverse, the triple composition $[\xi] \cdot[\eta] \cdot[\zeta]$ is well defined in $H_{p+q+r-2 n}\left(L M ; \mathbb{Z}_{o r}\right)$. Moreover, in that situation the following facts are easily checked:

$-\xi \times \eta$ is transverse;

- $(\xi * \eta) \times \zeta$ is transverse (where $*$ stands for the loop intersection product);

- $(\xi \cdot \eta) \cdot \zeta$ coincides with $\xi \cdot \eta \cdot \zeta$ up to a canonical reparametrization of the circle.

The last item yields the associativity in homology once it is observed that the same is true for other bracketing.

Now the only question is how to make $\xi \times \eta \times \zeta$ transverse when the is not. It is not sufficient to move one factor, as one factor in $M \times M \times M$ is not transverse to the small diagonal. It is necesary to move two factors, say $\xi \times \eta$, but keeping the product structure, that is moving through product chains $\xi^{t} \times \eta^{t}$; it is again transversality with constraint.

Remark 3.6. Each free loop $\gamma, \theta \mapsto \gamma(\theta)$, gives rise to a 1-cycle $\bar{\Delta}(\gamma)$ of loops by rotating the source:

$$
\bar{\Delta}(\gamma)(t)(\theta)=\gamma(t+\theta), t \in S^{1} .
$$

This map induces $\Delta: H_{*}(L M) \rightarrow H_{*+1}(L M)$, with twisted coefficients when $M$ is not orientable. Arguing in a similar way as in the previous proposition 3.5, one could prove the theorem of Chas-Sullivan that $\mathbb{H}_{*}(L M)$, endowed with the loop product and $\Delta$, is a BatalinVilkovsky algebra.

\section{A multiplicative SPeCtral SEQUence}

In this section, using our definition of the loop product, we discuss multiplicative properties which have been stated and proved by Mark Goresky and Nancy Hingston in [9], $\S 12$ (up to the coefficient system). The setting is the one that R. Bott first considered in his seminal paper [何, where he studied the standard $n$-sphere, and in the next paper [2]. We summarize his results as follows. Let $M$ be an $n$-dimensional closed Riemannian manifold whose primitive geodesics are all simple loops with the same length $(=1$, say). Denote by $\Lambda$ the space of loops parametrized proportionally to arc length. The class of regularity is not very important here. For instance take piecewise smooth loops; a good class is the Sobolev class $H^{1}\left(S^{1}, M\right)$. Let $\ell^{2}: \Lambda \rightarrow \mathbb{R}$ be the squared length. Bott proved that it is a nondegenerate function (now called a Morse-Bott function) and he calculated the index of the critical points (that is, the closed geodesics). For $p \in \mathbb{N}$, let $\Lambda_{p}$ be the subspace of loops of length $\leq p$ and $\Sigma_{p}$ be the space of geodesics of length $p$. A geodesic in $\Sigma_{p}$ is just a primitive geodesic which is traversed $p$ times. As a manifold $\Sigma_{0} \cong M$ and, for $p \geq 1, \Sigma_{p} \cong U M$ where $U M$ stands for the unit tangent space to $M$. Let 
$\alpha_{p}$ be the index of the Hessian of $\ell^{2}$ at any point of $\Sigma_{p}$; obviously $\alpha_{0}=0$. Bott proved the iteration formula:

$$
\alpha_{p}=p \alpha_{1}+(p-1)(n-1) .
$$

Moreover he calculated (with $\mathbb{Z}_{2}$ coefficients) the spectral sequence derived from the filtration $\Lambda_{0} \subset \Lambda_{1} \subset \ldots$ of $\Lambda$. We are going to consider the same spectral sequence, up to some regrading.

Definition 4.1. A spectral sequence $\left\{E_{p, q}^{r}, d^{r}\right\}_{r \geq 1}$ is said to be multiplicative when it is endowed with a product $E_{p, q}^{r} \otimes E_{p^{\prime}, q^{\prime}}^{r} \rightarrow E_{p+p^{\prime}, q+q^{\prime}}^{r}$ such that:

1) $d^{r}$ is a derivation in the graded sense:

$$
d^{r}(x \cdot y)=d^{r}(x) \cdot y+(-1)^{|x|} x \cdot d^{r}(y),
$$

where $|$.$| stands for the total degree |x|=p+q$ when $x \in E_{p, q}^{r}$;

2) the product on $E_{*, *}^{r+1} \cong H_{*}\left(E_{*, *}^{r}, d^{r}\right)$ is induced by the one of $E_{*, *}^{r}$ on the ideal ker $d^{r}$.

Following Chataur-Le Borgne in [5], we set

$$
\mathbb{E}_{p, q}^{1}=E_{p, q+n}^{1}=H_{p+q+n}\left(\Lambda_{p}, \Lambda_{p-1} ; e v_{0}^{*}\left(\mathbb{Z}_{o r}\right)\right) .
$$

The differential $d^{1}$ is the connecting homomorphism in the long exact sequence of the triple $\left(\Lambda_{p}, \Lambda_{p-1}, \Lambda_{p-2}\right)$. More generally, $d^{r}: E_{*, *}^{r} \rightarrow E_{*, *}^{r}$ is defined by the usual algorithm associated to the filtration $\cdots \Lambda_{p-1} \subset \Lambda_{p} \subset \Lambda_{p+1} \subset \cdots \Lambda$ (see [10], Chap. 2). Notice that page 0 exists in this spectral sequence, but there is no multiplicative structure on it.

Proposition 4.2. The loop product endows $\left\{\mathbb{E}_{*, *}^{r}\right\}_{r \geq 1}$ with a multiplicative structure.

Proof. Here the system of coefficients $e v_{0}^{*}\left(\mathbb{Z}_{o r}\right)$ is understood. We first look at the desired properties on page $r=1$. When $\xi$ is a relative $i$-cycle of the pair $\left(\Lambda_{p}, \Lambda_{p-1}\right), \eta$ is a relative $j$-cycle of the pair $\left(\Lambda_{p^{\prime}}, \Lambda_{p^{\prime}-1}\right)$ and $\xi \times \eta$ is transverse, the loop intersection product $\xi * \eta$ is a $(i+j-n)$-chain of composable loops. By performing the composition we get a chain $c$ of loops of length $\leq p+p^{\prime}$ since the length of the composed loop is just the sum of the lengths of the entries. The boundary of $c$ is a cycle in $\Lambda_{p+p^{\prime}-1}$. The class of $[\xi] \cdot[\eta]$ is well defined in $H_{i+j-n}\left(\Lambda_{p+p^{\prime}}, \Lambda_{p+p^{\prime}-1}\right)$. After regrading, this product behaves well with respect to the bi-degree. It remains to check that $d^{1}$ is a derivation.

We look first at a transverse bi-simplex $u \times v$ of bi-degree $(i, j)$ in $\Lambda \times \Lambda$. Let $W \subset \Delta^{i} \times \Delta^{j}$ be the preimage of the diagonal $\Delta_{M}$ by $e v_{0}(u) \times e v_{0}(v)$. Its boundary is made of two parts:

- $\partial_{1} W:=W \cap\left(\partial \Delta^{i} \times \Delta^{j}\right)$

- $\partial_{2} W:=W \cap\left(\Delta^{i} \times \partial \Delta^{j}\right)$

Each part gives rise to an $(i+j+n-1)$-chain in $\Lambda$ which is a part of $\partial(u \cdot v)$. As in the proof of lemma 2.9 the second chain is endowed with the sign $(-1)^{i}$ according to the formula for the total boundary which is written after definition 2.4. Taking the Dold sign into account yields:

$$
\partial(u \cdot v)=(\partial u) \cdot v+(-1)^{n-i} u \cdot(\partial v) .
$$

By summing such a formula over all bi-chains forming $\xi \times \eta$, we get:

$$
\partial(\xi \cdot \eta)=(\partial \xi) \cdot \eta+(-1)^{n-i} \xi \cdot(\partial \eta) .
$$


After regrading, it becomes a derivation formula. When $\xi$ and $\eta$ are relative cycles as above, $d^{1}([\xi] \cdot[\eta])=\left(d^{1}[\xi]\right) \cdot[\eta]+(-1)^{|\xi|}[\xi] \cdot d^{1}[\eta]$; hence, property 1 from definition 4.1 holds for $r=1$.

The product on $\mathbb{E}_{*, *}^{2}$ is defined by taking $\xi$, a relative cycle of the pair $\left(\Lambda_{p}, \Lambda_{p-2}\right)$, and $\eta$, a relative cycle in the pair $\left(\Lambda_{p^{\prime}}, \Lambda_{p^{\prime}-2}\right)$, where $\xi \times \eta$ is transverse. Thus the chain of composed loops is a relative cycle of the pair $\left(\Lambda_{p+p^{\prime}}, \Lambda_{p+p^{\prime}-2}\right)$. It can be checked that elements in ker $d^{1}$ are represesentable by such cycles. Hence property 2 from definition 4 .1 holds for $r=1$.

The same argument applies for every $r \geq 1$ once one remembers the definition of $E_{p, *}^{r}$ associated to the filtration of $\Lambda$. The product on $\mathbb{E}_{*, *}^{r}$ is defined by taking $\xi$, a relative cycle of the pair $\left(\Lambda_{p}, \Lambda_{p-r}\right)$, and $\eta$, a relative cycle of the pair $\left(\Lambda_{p^{\prime}}, \lambda_{p^{\prime}-r}\right)$, where $\xi \times \eta$ is transverse. The boundary operator $d^{r}$ is induced by $\partial$ and is not affected by the value of $r$. After regrading $d^{r}$, like $d^{1}$, is a derivation and the product which is induced on its homology is the one of $E_{*, *}^{r+1}$. $\diamond$

\subsection{The Thom isomorphism.}

There is a Morse-Bott version of the famous Morse lemma. It yields a "normal" form for a Morse-Bott function near a critical manifold. We are going to apply it to the function $\ell^{2}: \Lambda \rightarrow \mathbb{R}$ near the critical manifold $\Sigma_{p}$ whose index is $\alpha_{p}$. If one feels uncomfortable by applying this lemma in infinite dimension, one can take a finite dimensional approximation of $\Lambda$ near $\Sigma_{p}$ by considering the space of geodesics polygons (still parametrized proportionally to arc length) with $N p$ edges of equal length, where $1 / N$ is less than the injectivity radius. Let $E_{p}^{-}$be the vector bundle of rank $\alpha_{p}$ over $\Sigma_{p}$ generated by the eigenvectors of the Hessian of $\ell^{2}$ (with respect to some Riemannian metric on $\Lambda$ ) whose eigenvalues are negative; it is a sub-bundle of $T \Lambda \mid \Sigma_{p}$. With notation borrowed from [9], let $\Sigma_{p}^{-}=\exp \left(E_{p}^{-}\right)$denote the unstable manifold of $\Sigma_{p}$ with respect to the gradient of $\ell^{2}$; we are mainly interested in its germ along the critical manifold. Finally $\Lambda_{p}^{-}$denotes the open set of loops whose length is less than $p$. We have $\Sigma_{p}^{-} \backslash \Sigma_{p} \subset \Lambda_{p}^{-}$(as a consequence of Taylor expansion).

The Morse-Bott lemma states:

(1) $\Lambda_{p}^{-}$retracts by deformation onto $\Lambda_{p-1}$;

(2) $\Lambda_{p}$ retracts by deformation onto $\Lambda_{p-1} \cup \Sigma_{p}^{-}$.

As a consequence, the inclusions of pairs induce the following isomorphisms:

$$
H_{*}\left(\Lambda_{p}, \Lambda_{p-1}\right) \cong H_{*}\left(\Lambda_{p}, \Lambda_{p}^{-}\right) \cong H_{*}\left(\Sigma_{p}^{-}, \Sigma_{p}^{-} \backslash \Sigma_{p}\right) .
$$

Here the system of coefficients is $e v_{0}^{*}\left(\mathbb{Z}_{o r}\right)$. The orientation of the fibre bundle $E_{p}^{-}$being twisted as $e v_{0}^{*}(\mathbb{Z})$ is (see [9] Prop. 12.2), we are ready to apply the Thom isomorphism:

$$
h_{p}: H_{*}\left(\Sigma_{p} ; \mathbb{Z}\right) \cong H_{*+\alpha_{p}}\left(\Sigma_{p}^{-}, \Sigma_{p}^{-} \backslash \Sigma_{p} ; e v_{0}^{*}\left(\mathbb{Z}_{o r}\right)\right),
$$

which can also be read, when $p>0$,

$$
h_{p}: H_{*}(U M ; \mathbb{Z}) \cong H_{*+\alpha_{p}}\left(\Lambda_{p}, \Lambda_{p-1} ; e v_{0}^{*}\left(\mathbb{Z}_{o r}\right)\right) .
$$

Notice also that the Gysin morphism

$$
H_{*}\left(M ; \mathbb{Z}_{\text {or }}\right) \rightarrow H_{*+n-1}(U M ; \mathbb{Z})
$$


makes $H_{*}(U M ; \mathbb{Z})$ a $H_{*}\left(M ; \mathbb{Z}_{\text {or }}\right)$-module for the intersection product. In the next proposition the coefficients are not written; they are meant as we just specified them.

Proposition 4.4. (Goresky-Hingston, [9] Theorem 12.5). The Thom isomorphisms carry the intersection product of $H_{*}(U M)$ into the loop product of $\oplus_{p>0} H_{*+\alpha_{p}}\left(\Lambda_{p}, \Lambda_{p-1}\right)$. Moreover, they carry the $H_{*}(M)$-module structure of $H_{*}(U M)$ to the $H_{*}\left(\Lambda_{0}\right)$-module structure of $H_{*}\left(\Lambda_{p}, \Lambda_{p-1}\right)$.

The proof below mainly follows the same line as [9]. But it is based on the notion of loop product that we have introduced in the previous section.

Proof. There are several steps.

A) One can factorize the intersection product int of $H_{*}(U M)$ in the following way:

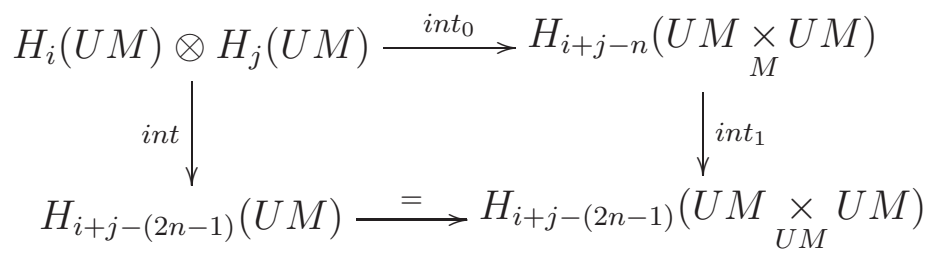

Indeed, starting with a transverse bi-cycle $\xi \times \eta$ we may first intersect it with the fibered product $U M \underset{M}{\times} U M \subset U M \times U M$ yielding an $(i+j-n)$-cycle $\zeta$ (this induces the morphism $i_{0} t_{0}$ ), which, in turn, is transverse to the diagonal $\Delta_{U M}=U M \underset{U M}{\times} U M$ (this induces the morphism int $\left._{1}\right)$.

B) Since $e v_{0} \mid \Sigma_{p}$ is a smooth submersion, the fibered product $\Sigma_{p, p^{\prime}}:=\Sigma_{p} \underset{M}{\times} \Sigma_{p^{\prime}}$ is a smooth manifold. A point of it is a pair of closed geodesics of respective lengths $p$ and $p^{\prime}$ which are composable as loops. In general the composed loop is not a geodesic.

Similarly, since $\Sigma_{p}^{-}$is tangent to $E_{p}^{-}$, a fibre bundle over $\Sigma_{p}$, then $e v_{0} \mid \Sigma_{p}^{-}$is also a submersion onto $M$ near $\Sigma_{p}$. Thus $\Sigma_{p, p^{\prime}}^{-}:=\Sigma_{p}^{-} \underset{M}{\times} \Sigma_{p^{\prime}}^{-}$is smooth near $\Sigma_{p, p^{\prime}}$. The tangent space to $\Sigma_{p, p^{\prime}}^{-}$along $\Sigma_{p, p^{\prime}}$ is the restriction of $E_{p}^{-} \times E_{p^{\prime}}^{-}$, a vector bundle of rank $\alpha_{p}+\alpha_{p^{\prime}}$. So we have a Thom isomorphism:

$$
h_{p, p^{\prime}}: H_{*}\left(\Sigma_{p, p^{\prime}}\right) \cong H_{*+\alpha_{p}+\alpha_{p^{\prime}}}\left(\Sigma_{p, p^{\prime}}^{-}, \Sigma_{p, p^{\prime}}^{-} \backslash \Sigma_{p, p^{\prime}}\right) .
$$

At the chain level of small simplices, the Thom isomorphism is generated by the following Thom "extension": take a small simplex in the base of a disk bundle (which hence is trivial over the considered small simplex) and cross it with the fiber. If $\xi \times \eta$ is a bi-cycle in $\Sigma_{p} \times \Sigma_{p^{\prime}}$ transverse to $\Sigma_{p, p^{\prime}}$, then the Thom extension $\tilde{\xi} \times \tilde{\eta}$ is transverse to $\Sigma_{p, p^{\prime}}^{-}$and its intersection with $\Sigma_{p, p^{\prime}}^{-}$is the Thom extension of the intersection cycle in the base $\Sigma_{p, p^{\prime}}$. This proves that the Thom isomorphism carries the loop intersection product $H_{i}\left(\Sigma_{p}\right) \otimes H_{j}\left(\Sigma_{p^{\prime}}\right) \rightarrow H_{i+j-n}\left(\Sigma_{p, p^{\prime}}\right)$ to the suitable relative version of the loop intersection product $H_{i+\alpha_{p}}\left(\Sigma_{p}^{-}\right) \otimes H_{j+\alpha_{p^{\prime}}}\left(\Sigma_{p^{\prime}}^{-}\right) \rightarrow H_{i+j+\alpha_{p}+\alpha_{p^{\prime}}-n}\left(\Sigma_{p, p^{\prime}}^{-}\right)$. Note that, after identification, the first morphism is nothing but int $_{0}$ from A).

C) We observe that $\Sigma_{p+p^{\prime}}$ lifts (by a section of the composition map) as a submanifold of codimension $n-1$ in $\Sigma_{p, p^{\prime}}$. Indeed, any smooth geodesic of length $p+p^{\prime}$ splits uniquely into two geodesics of respective lengths $p$ and $p^{\prime}$. Conversely, $\left(\gamma, \gamma^{\prime}\right) \in \Sigma_{p, p^{\prime}}$ belongs to this lifting 
of $\Sigma_{p+p^{\prime}}$ if and only if the initial velocities $\dot{\gamma}(0)$ and $\dot{\gamma}^{\prime}(0)$ are positively proportional, which is a condition of codimension $n-1$. Thus, the composition is a map of pairs:

$$
\text { comp : }\left(\Sigma_{p, p^{\prime}}^{-}, \Sigma_{p, p^{\prime}}^{-} \backslash \Sigma_{p+p^{\prime}}\right) \rightarrow\left(\Lambda_{p+p^{\prime}}, \Lambda_{p+p^{\prime}} \backslash \Sigma_{p+p^{\prime}}\right) .
$$

The normal bundle to $\Sigma_{p+p^{\prime}}$ in $\Sigma_{p, p^{\prime}}^{-}$, denoted by $\nu$, is the direct sum $E_{p}^{-} \oplus E_{p^{\prime}}^{-} \oplus \nu_{p, p^{\prime}}$ where $\nu_{p, p^{\prime}}$ denotes the normal bundle to $\Sigma_{p+p^{\prime}}$ in $\Sigma_{p, p^{\prime}}$. The rank of $\nu$ is $\alpha_{p}+\alpha_{p^{\prime}}+n-1$. According to Bott's iteration formula, it equals $\alpha_{p+p^{\prime}}$, which is the rank of $E_{p+p^{\prime}}^{-}$.

If $\xi$ is a relative cycle in $\left(\Sigma_{p, p^{\prime}}^{-}, \Sigma_{p, p^{\prime}}^{-} \backslash \Sigma_{p, p^{\prime}}\right)$ transverse to $\Sigma_{p+p^{\prime}}$, its trace in the pair $\left(\Sigma_{p, p^{\prime}}^{-}, \Sigma_{p, p^{\prime}}^{-} \backslash \Sigma_{p+p^{\prime}}\right)$ is the Thom extension of its intersection with $\Sigma_{p+p^{\prime}}$. In other words we have the following commutative diagram:

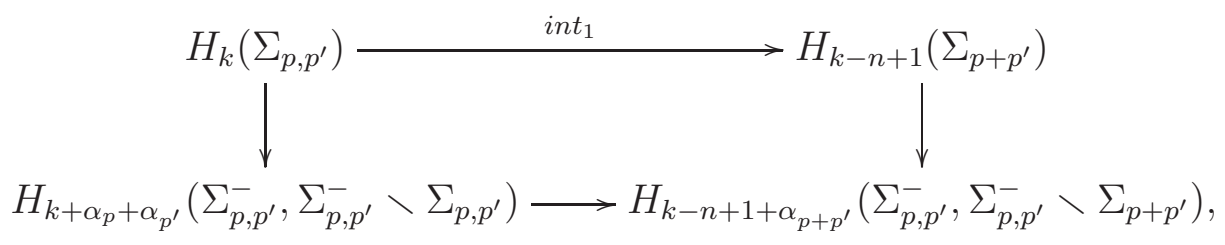

where the vertical arrows are the respective Thom isomorphisms. The proof of proposition 4.4 is not yet achieved because we are still at the level of composable loops and the composition has not yet been performed.

D) Some difficulty comes here from the fact that the composition map comp : $\Sigma_{p, p^{\prime}}^{-} \rightarrow \Lambda_{p+p^{\prime}}$ could be singular along $\Sigma_{p+p^{\prime}}$ in the direction of $\nu_{p, p^{\prime}}$ since comp maps $\Sigma_{p, p^{\prime}}$ into the critical level set of $\ell$ whose value is $p+p^{\prime}$. We are going to construct:

a) a linear embedding $\varphi: \nu \rightarrow T \Lambda \mid \Sigma_{p+p^{\prime}}$, over the identity of $\Sigma_{p+p^{\prime}}$, such that $\operatorname{Hess}\left(\ell^{2}\right) \circ \varphi$ is negative definite;

b) a homotopy from comp to $\exp \circ \varphi \circ \mathrm{exp}^{-1}$ among the maps of pairs

$$
\left(\Sigma_{p, p^{\prime}}^{-}, \Sigma_{p, p^{\prime}}^{-} \backslash \Sigma_{p+p^{\prime}}\right) \rightarrow\left(\Lambda_{p+p^{\prime}}, \Lambda_{p+p^{\prime}} \backslash \Sigma_{p+p^{\prime}}\right) .
$$

If such items exist, $\operatorname{comp}_{*}: H_{*}\left(\Sigma_{p, p^{\prime}}^{-}, \Sigma_{p, p^{\prime}}^{-} \backslash \Sigma_{p+p^{\prime}}\right) \rightarrow H_{*}\left(\Lambda_{p+p^{\prime}}, \Lambda_{p+p^{\prime}} \backslash \Sigma_{p+p^{\prime}}\right)$ commutes with the respective Thom isomorphisms, as it is true for $\varphi_{*}$, and the proof of proposition 4.4 is finished.

We choose $\varphi$ to be the identity of the factor $E_{p}^{-} \oplus E_{p^{\prime}}^{-}$. This makes sense as $\left(E_{p}^{-}\right)_{\gamma}$ (resp. $\left.\left(E_{p}^{\prime-}\right)_{\gamma^{\prime}}\right)$ embeds canonically into $\left(T \Lambda_{p+p^{\prime}}\right)_{\gamma * \gamma^{\prime}}$, thought of as the set of vector fields tangent to $M$ along $\gamma * \gamma^{\prime}$ : it is a set of tangent vector fields which are vanishing along $\gamma^{\prime}$ (resp. along $\gamma$ ); notice that a vector in $\left(E_{p}\right)_{\gamma}$ corresponds to a vector field along $\gamma$ which vanishes at $\gamma(0)$ since $U M$ is homogeneous.

Let $\left(\gamma, \gamma^{\prime}\right) \in \Sigma_{p+p^{\prime}} \subset \Sigma_{p, p^{\prime}}$ and $X$ be a vector in $\nu_{\left(\gamma, \gamma^{\prime}\right)}$. This vector indicates an infinitesimal deformation of $\left(\gamma, \gamma^{\prime}\right)$ among the pairs of composable closed smooth geodesics, deformation which separates the directions of their initial velocities. Precisely, there is a one-parameter family $\left(\gamma_{u}, \gamma_{u}^{\prime}\right), u \in[0, \varepsilon)$, of pairs of closed geodesics such that $\gamma_{u}(0)=\gamma(0)=\gamma^{\prime}(0)=\gamma_{u}^{\prime}(0)$ and $\frac{d}{d u}\left(\dot{\gamma}_{u}(0)-\dot{\gamma}_{u}^{\prime}(0)\right)_{\mid u=0}=X$ (up to a positive scalar); here we identify $\nu_{\left(\gamma, \gamma^{\prime}\right)}$ with the fibre $U M_{\gamma(0)}$. Instead of taking this deformation which leaves the length unchanged, we consider the following shortening deformation made of geodesic triangles $T_{u}$ : the first edge in $T_{u}$ is $\gamma_{u}(t), t \in[0,1-\varepsilon]$, the second edge joins geodesically the point $\gamma_{u}(1-\varepsilon)$ to the nearby point 
$\gamma_{u}^{\prime}(\varepsilon)$ and the third edge is $\gamma_{u}^{\prime}(t), t \in[\varepsilon, 1]$ (the triangle is parametrized proportionally to arc length). We define $\varphi(X)$ to be the infinitesimal generator of this family. By estimating $\ell\left(T_{u}\right)$ it is easily seen that $\operatorname{Hess}\left(\ell^{2}\right)(\varphi(X))<0$. Moreover $\varphi(X)$ is independent of $E_{p}^{-} \oplus E_{p^{\prime}}^{-}$since, as a tangent vector field to $M$ along the composed loop $\gamma * \gamma^{\prime}$, it does not vanish at the junction point $\gamma(1)=\gamma^{\prime}(0)$. This proves item a). In order to obtain item b) it is sufficient to make $\epsilon$ tend to 0 .

By taking the different regradings into account, the Thom isomorphism yields when $p>0$ :

$$
\mathbb{E}_{p, q}^{1} \cong H_{p+q+n-\alpha_{p}}\left(\Sigma_{p}, \mathbb{Z}\right) \cong H_{p+q+n-\alpha_{p}}(U M, \mathbb{Z}) \cong \mathbb{H}_{p+q+n-\alpha_{p}-(2 n-1)}(U M, \mathbb{Z}),
$$

where $\mathbb{H}_{*}(U M ; \mathbb{Z})$ denotes the regraded intersection ring of the $(2 n-1)$-dimensional manifold $U M$. Similarly, after regrading the Gysin morphism, $H_{*}\left(M ; \mathbb{Z}_{\text {or }}\right) \rightarrow H_{*+n-1}(U M ; \mathbb{Z})$, becomes a morphism of degree $0, \mathbb{H}_{*}\left(M ; \mathbb{Z}_{\text {or }}\right) \rightarrow \mathbb{H}_{*}(U M ; \mathbb{Z})$, making $\mathbb{H}_{*}(U M ; \mathbb{Z})$ a $\mathbb{H}_{*}\left(M ; \mathbb{Z}_{\text {or }}\right)$-module for the intersection product.

The multiplicative structure on $\mathbb{E}_{*, *}^{1}$ can be interpreted in terms of $\mathbb{H}_{*}(U M)$. The following statement is due to Chataur-Le Borgne in [5] (up to the coefficient system).

\section{Proposition 4.5.}

1) There is an isomorphism of bigraded rings:

$$
\mathbb{E}_{*, *}^{1} \cong \mathbb{H}_{*}\left(M ; \mathbb{Z}_{\text {or }}\right) \oplus \mathbb{H}_{*}(U M ; \mathbb{Z})[T]_{\geq 1}
$$

Here $\mathbb{E}_{*, *}^{1}$ is endowed with the bigraded ring structure yielded by proposition 4.9 . The intersection rings $\mathbb{H}_{*}(M)$ and $\mathbb{H}_{*}(U M)$ have bi-degree $(0, *)$. The new variable $T$ has bi-degree $\left(1, \alpha_{1}+n-2\right)$ and appears only at a positive power. Regarding $\mathbb{H}_{*}(U M)$ as a $\mathbb{H}_{*}(M)$-module, the right hand side has a well-defined ring structure.

2) The differential $d^{1}$ on page 1 vanishes at every place.

3) The page $\infty$ inherits the same isomorphism of bigraded rings as page 1:

$$
\mathbb{E}_{*, *}^{\infty} \cong \mathbb{H}_{*}\left(M ; \mathbb{Z}_{\text {or }}\right) \oplus \mathbb{H}_{*}(U M ; \mathbb{Z})[T]_{\geq 1} .
$$

Proof. 1) We read the first page of the spectral sequence, $\mathbb{E}_{*, *}^{1}$, via the Thom isomorphism taking proposition 4.4 into account. For instance, we have (without writing the coefficients):

$$
\mathbb{H}_{0}(U M)=H_{2 n-1}(U M)=H_{2 n-1+\alpha_{1}}(U M) \cong E_{1,2 n-2+\alpha_{1}}^{1}=\mathbb{E}_{1, n-2+\alpha_{1}}^{1}
$$

and $T$ is nothing but the image of the unit $\mu \in \mathbb{H}_{0}(U M)$ (that is, the fundamental class of $U M)$ through the Thom isomorphism. Once the desired ring isomorphism is specified on $T$ it extends globally using the multiplicative property of the Thom isomorphism.

2) $\operatorname{As} \mathbb{H}_{*}\left(\Lambda_{0}\right)$ is a direct factor in $\mathbb{H}_{*}(\Lambda), d^{1}: \mathbb{E}_{1, *}^{1} \rightarrow \mathbb{E}_{0, *}^{1}$ has to vanish. In particular, $d^{1}(T)=0$. As $d^{1}$ is a derivation (prop. 4.2), it vanishes everywhere.

3) As a consequence, page 2 of the spectral sequence is isomorphic to page 1 as a bi-graded ring. Therefore the differential $d^{2}$ vanishes for the same reason as $d^{1}$. Proceeding recursively through the successive pages yields the conclusion. 


\section{REFERENCES}

[1] R. Bott, Nondegenerate critical manifolds, Annals of Math. 60 (1954), 248-261.

[2] R. Bott, On manifolds all of whose geodesics are closed, Annals of Math. 60 (1954), 375-382.

[3] E. Castillo, R. Diaz, Homology and manifolds with corners, Affrican Diaspora J. of Math. 8, 2 (2009), $100-11$.

[4] M. Chas, D. Sullivan, String Topology, ArXiv:math.GT/9911159.

[5] D. Chataur, J.-F. Le Borgne, Homology of spaces of regular loops in the sphere, ArXiv:math.AT/0811.3319.

[6] R. Cohen, J. Jones, A homotopy theoretic realization of string topology, Math. Ann. 324 (2002), $773-798$.

[7] A. Dold, Lectures on Algebraic Topology, Grundlehren Math. 200, Springer, 1972.

[8] Y. Félix, J.-C. Thomas, String topology on Gorenstein spaces, Math. Ann. 345 (2009), 417-452.

[9] M. Goresky, N. Hingston, Loop Products and Closed Geodesics, Duke Math. J. 150, 1 (2009), $117-209$.

[10] J. McCleary, User's Guide to Spectral Sequences, Secon edition, Cambridge Univ. Press, 2001.

[11] J.E. McClure, On the chain-level intersection pairing for PL manifolds, Geometry \& Topology 10 (2006), 1391-1424.

[12] R. Thom, Les singularités des applications différentiables, Ann. Inst. Fourier, Grenoble 6 (1955-1956), 43-87.

[13] J.H.C. Whitehead, On $C^{1}$-complexes. Ann. of Math. (2) 41, (1940), 809-824.

Laboratoire de mathématiques JeAn Leray, UMR 6629 Du CNRS, Faculté Des Sciences et Techniques, Université de Nantes, 2, rue de la Houssinière, F-44322 Nantes Cedex 3, France. E-mail address: francois.laudenbach@univ-nantes.fr 